\title{
AN EXAMPLE ON EMBEDDING UP TO HOMOTOPY TYPE
}

\section{ELMER REES ${ }^{1}$}

Abstract. A finite complex $K$ is constructed with the following property. $K \bigvee S^{r}$ embeds in $R^{n}$ up to homotopy type but $K$ does not.

T. Ganea (see $[4$, p. 156]) has asked the following

QUestion. If $K$ is a finite complex dominated by a subset of $R^{n}$, is there a subcomplex of $R^{n}$ of the homotopy type of $K$ ?

In this note this is answered negatively. A finite complex $K_{n}$ of dimension $8 n-3$ is constructed (for each $n \geqq 3$ ) which cannot be embedded up to homotopy type in $S^{8 n}$; however, there is a subcomplex of $S^{8 n}$ homotopically equivalent to $K_{n} \bigvee S^{4 n}$.

Consider the complex Stiefel manifold $W_{2 n, 2}$ of two frames in $\boldsymbol{C}^{2 n}$. $W_{2 n, 2}$ has a cell decomposition $\left(S^{4 n-3} \bigvee S^{4 n-1}\right) \cup_{\alpha_{n}} e^{8 n-4}$ where $\alpha_{n}$ $=i_{4 n-3} \circ \beta_{n}+\left[i_{4 n-3}, i_{4 n-1}\right]\left(i_{m}\right.$ denotes the inclusion of $\left.S^{m}\right)$. Its suspension $S W_{2 n, 2}$ is homotopically equivalent to $K_{n} \vee S^{4 n}$ where $K_{n}$ is $S^{4 n-2} \cup_{S \beta_{n}} e^{8 n-3}$ and $S^{2} \beta_{n}=-\left[\iota_{4 n-1}, \iota_{4 n-1}\right] \in \pi_{8 n-3} S^{4 n-1}$. All this is contained in [3].

Proposition 1. $K_{n}$ does not embed up to homotopy type in $S^{8 n}$ if $n \geqq 3$.

Proof. If $K_{n}$ does embed in $S^{8 n}$, it has complement homotopically equivalent to $S^{2} \cup_{\gamma_{n}} e^{4 n+1}$ and $S^{4 n-3} \gamma_{n}=S^{2} \beta_{n}=-\left[\iota_{4 n-1}, \iota_{4 n-1}\right]$ by the results of [2]. But by Corollary 1.3 of [1], if $n \geqq 3$ then [ $\iota_{4 n-1}, \iota_{4 n-1}$ ] is not a $(4 n-3)$-fold suspension.

Proposition 2. $W_{2 n, 2}$ embeds in $S^{8 n-1}$.

Proof. $W_{2 n, 2}$ is the sphere bundle of an $R^{4 n-2}$ bundle $\xi$ over $S^{4 n-1}$ and $\xi \oplus \epsilon^{2}$ is trivial. So $W_{2 n, 2}$ is included in the total space of $\epsilon^{4 n}$ which embeds in $S^{8 n-1}$.

Corollary. $K_{n} \vee S^{4 n}$ embeds in $S^{8 n}$.

Proof. $W_{2 n, 2}$ embeds in $S^{8 n-1}$ and so its suspension (which is homotopically equivalent to $K_{n} \bigvee S^{4 n}$, by the above) embeds in $S^{8 n}$.

Received by the editors November 30, 1969.

AMS subject classifications. Primary 5570.

Key words and phrases. Embedding up to homotopy type, complex Stiefel manifolds.

${ }^{1}$ Supported in part by National Science Foundation grant GP-7952X1. 
Therefore the complexes $K_{n}$ have the required properties.

\section{REFERENCES}

1. J. F. Adams, Vector fields on spheres, Ann. of Math. (2) 75 (1962), 603-632. MR $25 \# 2614$.

2. G. Cooke, Embedding certain complexes up to homotopy type in euclidean space, Ann. of Math. (2) 90 (1969), 144-156.

3. I. M. James and J. H. C. Whitehead, The homotopy theory of sphere bundles over spheres. I, Proc. London Math. Soc. (3) 4 (1954), 196-218. MR 15, 892.

4. S. P. Novikov, The topology summer institute (Seattle, Wash., 1963), Uspehi Mat. Nauk 20 (1965), no. 1 (121), 147-169=Russian Math. Surveys 20 (1965), no. $1,145-167$.

Institute for Advanced Study, Princeton, New Jersey 08540. 